\title{
An unusual reversible color change in a gastric lesion during real-time endoscopy
}

\author{
Shozo Osera ${ }^{1}$, Akihisa Tomori ${ }^{1}$, Aiko Arakawa ${ }^{1}$, Satoshi Shiozawa ${ }^{1}$, and Takeshi Hisa ${ }^{1}$ \\ ${ }^{1}$ Saku Central Hospital Advanced Care Center
}

July 22, 2020

\begin{abstract}
This report presents real-time endoscopic observations of an unusual flat gastric lesion that would appear upon inflation of the stomach and disappear upon deflation of the stomach. Although there are some reports regarding the evaluation of gastric blood flow, little has been reported about real-time color changes in gastric lesions.
\end{abstract}

\section{Key Clinical Message}

During endoscopy, a light purple flat lesion suddenly appeared at the antrum and the lesion disappeared subsequently. This is an unusual reversible color change that is associated with blood flow, and that must be differentiated from neoplasia.

A 63-year-old man with heart failure underwent esophagogastroduodenoscopy. The patient had not receivedHelicobacter pylori eradication therapy and results of serum antibody test were negative. Endoscopy revealed no gastric lesion upon initial scope insertion (Figure 1A ). During observation, an approximately 25-mm diameter, light-purple-colored flat lesion appeared at the antrum's greater curvature (Figure 1B, 1C ). Magnifying narrow-band imaging revealed a regular microsurface and microvascular pattern (Figure 1D ). The lesion subsequently faded upon slight deflation of the stomach (Figure 1E ), but reappeared upon reinflation (Figure 1F ).

The lesion was well-demarcated and flat. Biopsies of both the lesion and surrounding mucosa were performed to rule out neoplasm. Hematoxylin-and-eosin-staining of the lesion revealed mostly normal fundic glands; however, the gland density and mucosal thickness were slightly decreased compared to the surrounding mucosa (Figure 2 ). CD31 staining revealed little remarkable findings of vascular density. Computed tomography revealed no masses compressing gastric walls. Although there have been previous reports on evaluation of gastric blood flow, ${ }^{\mathbf{1 , 2}}$ investigations on real-time color changes in gastric lesions are rare. This study shows unusual reversible color change in a gastric lesion associated with slight blood flow changes and reflecting a small focal atrophic change which must be differentiated from neoplastic lesions.

\section{Author contribution}

Shozo Osera: conception and design, acquisition of data, analysis and interpretation of data, and manuscript drafting; Akihisa Tomori: analysis and interpretation of data, and manuscript revising; Aiko Arakawa: analysis and interpretation of data, and manuscript revising; Satoshi Shiozawa: acquisition of data, analysis and interpretation, and manuscript revising; Takeshi Hisa: manuscript revising. All authors approved the final version to be published, and agree to be accountable for all aspects of the work.

Informed consent : Informed consent was obtained from the patient for the publication of their information and imaging. 


\section{Conflict of interest : None}

Funding: This research did not receive any specific grant from funding agencies in the public, commercial, or not-for-profit sectors.

\section{References}

1. Berge ST, Safi N, Medhus AW, et al. Gastroscopy assisted laser Doppler flowmetry and visible light spectroscopy in patients with chronic mesenteric ischemia. Scand J Clin Lab Inv 2019;79:541-549.

2. Iwao T, Toyonaga A, Ikegami M, et al. Observer agreement and variability in measuring gastric mucosal blood flow by laser Doppler flowmetry in humans. Endoscopy 1993;25:274-277.

\section{Figure legends}

Figure 1 Endoscopic images

(A) No lesion upon the initial insertion of the scope into the stomach.

(B) (C) Sudden appearance of a light-purple, flat lesion at the greater curvature of the antrum.

(D) Small round pit seen upon magnification of narrow-band imaging.

(E) Subsequent fading of the lesion.

(F) The lesion reappeared upon sufficient inflation of the stomach.

Figure 2 Hematoxylin-and-eosin-stained section

The gland density and mucosal thickness of a part of the lesion were slightly decreased compared with those of the surrounding mucosa.

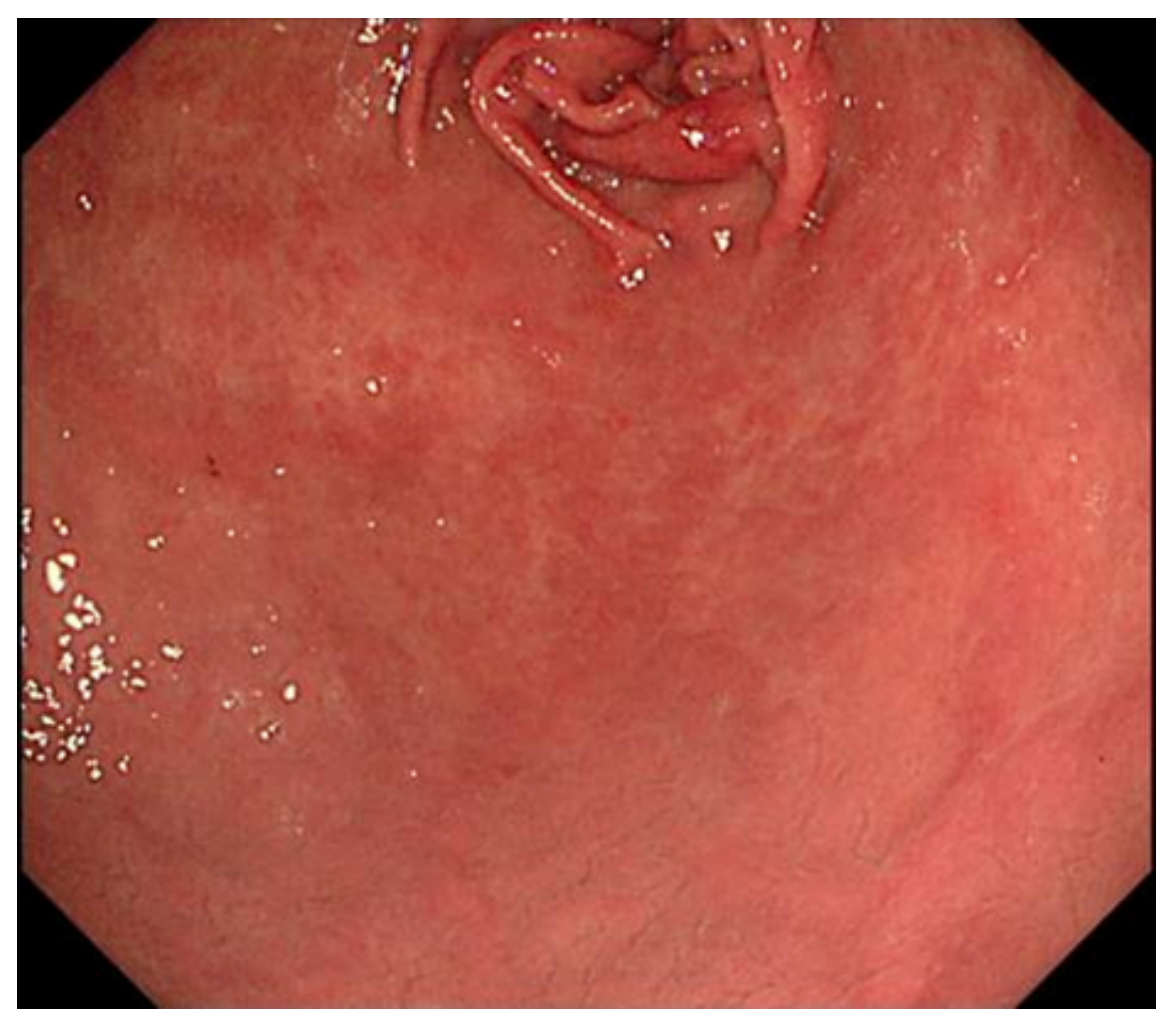



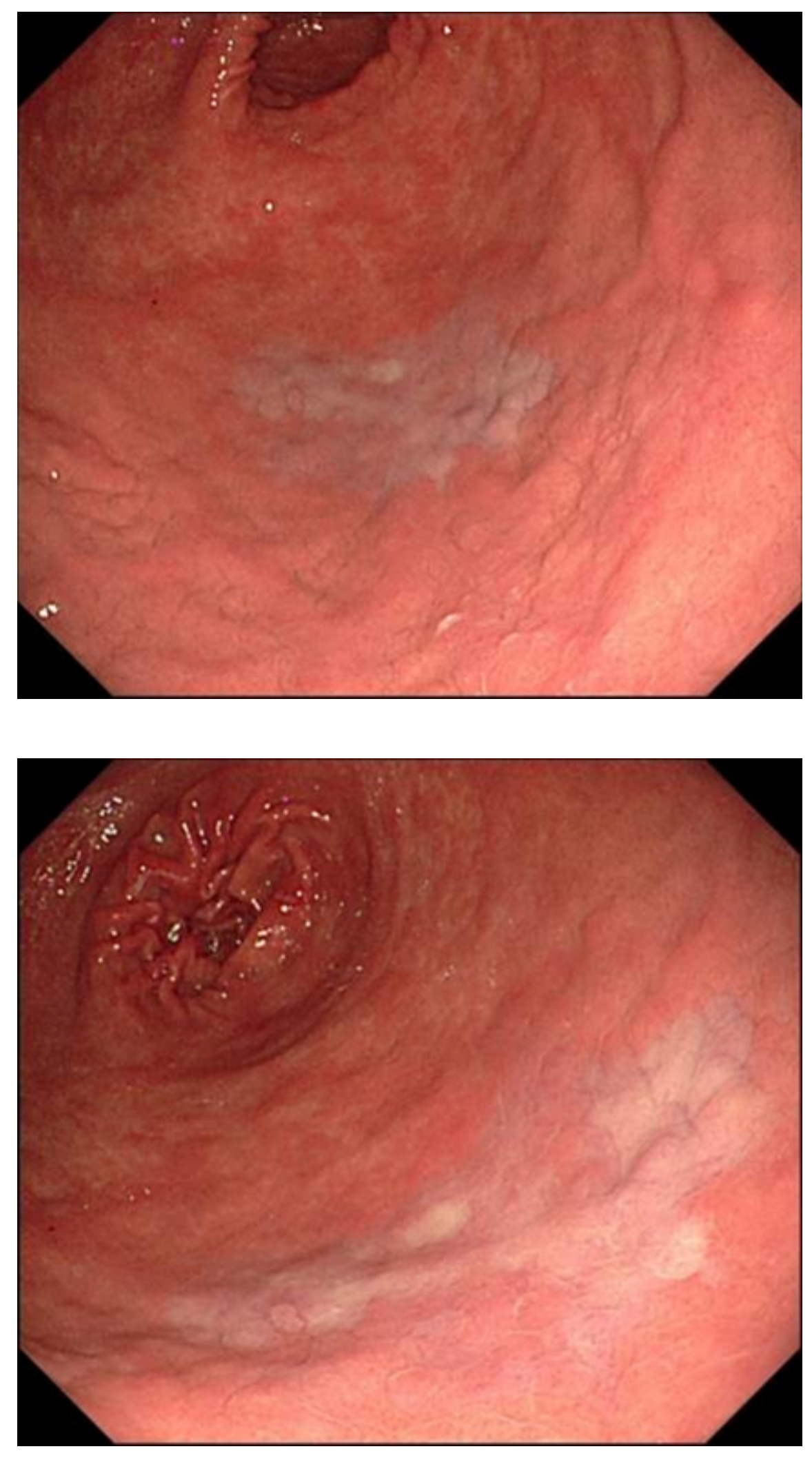

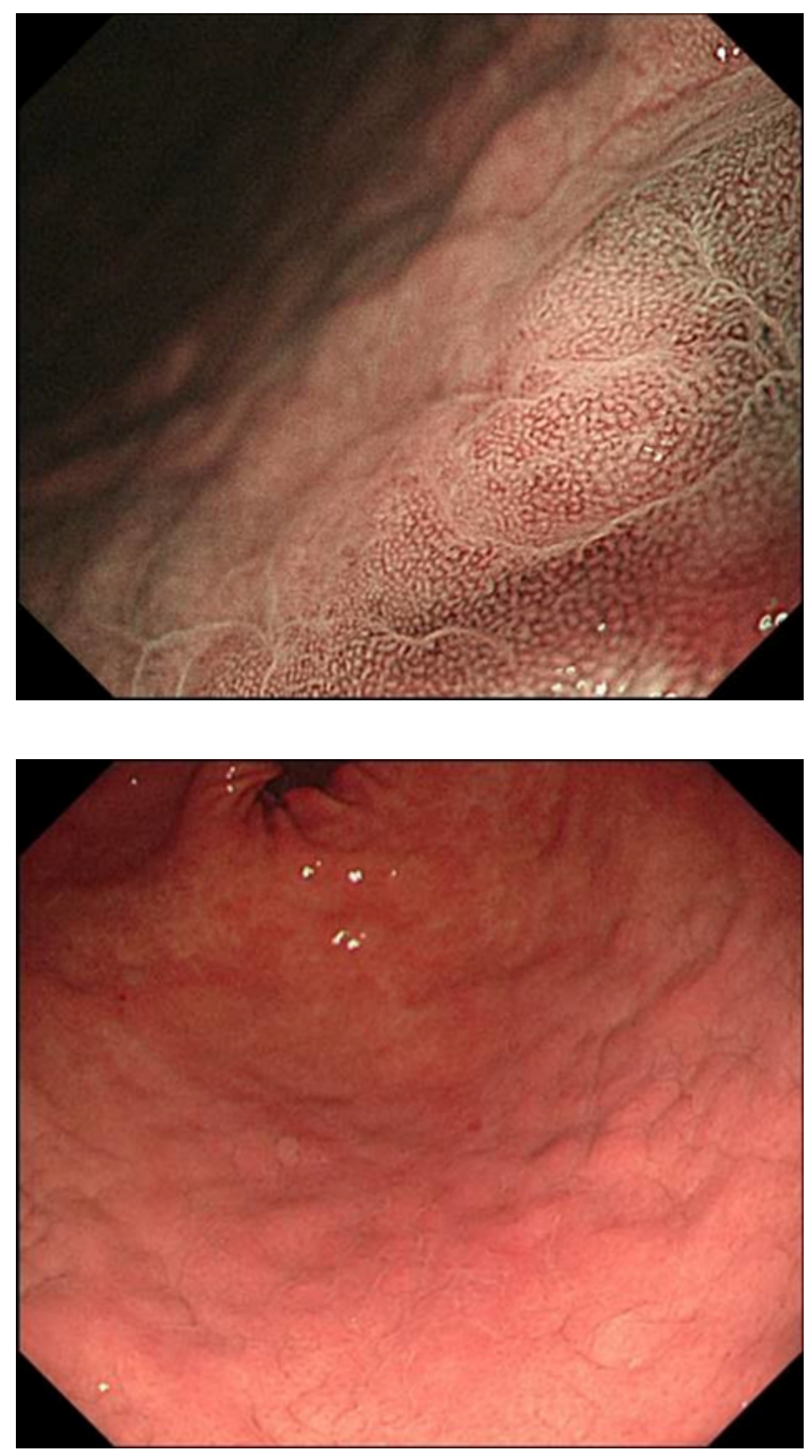

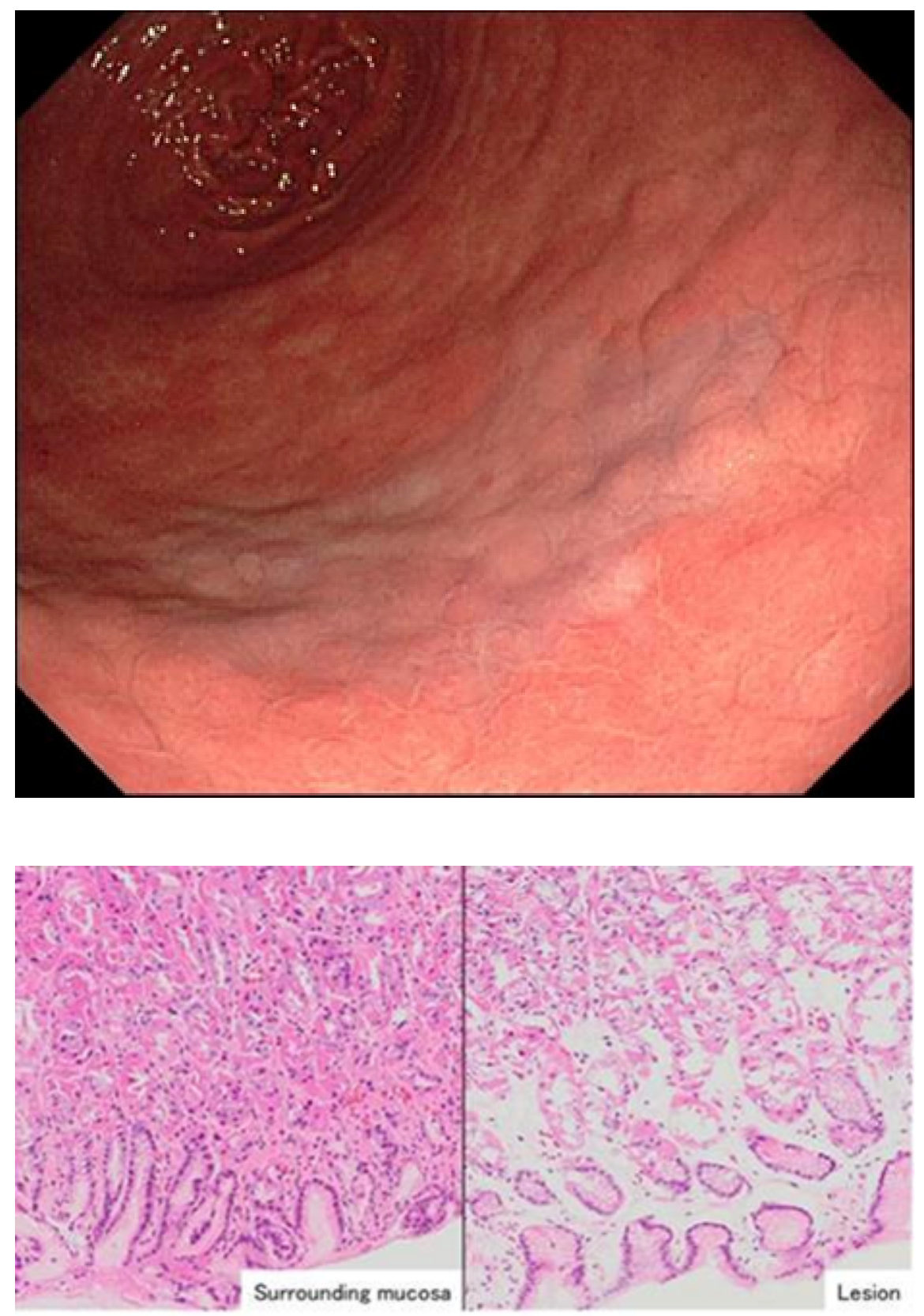